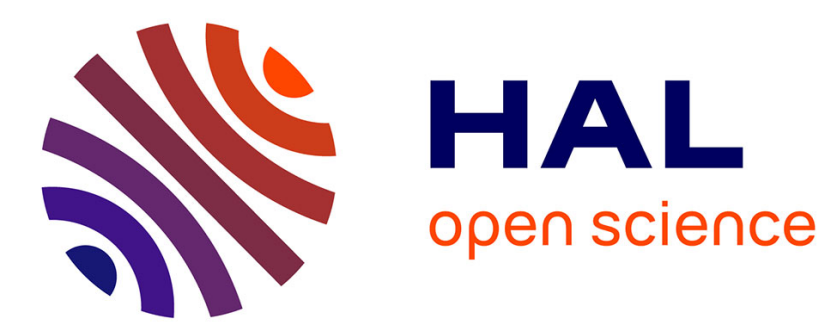

\title{
Euripide revisité dans Le Mystère d'Alceste de Marguerite Yourcenar
}

Rémy Poignault

\section{To cite this version:}

Rémy Poignault. Euripide revisité dans Le Mystère d'Alceste de Marguerite Yourcenar. O livro do tempo: escritas e reescritas: teatro greco-latino e sua recepção, II, Sousa e Silva, Maria de Fátima; Fialho, Maria do Céu Grácio Zambujo; Brandão, José Luís Lopes (éd.), Imprensa da Universidade de Coimbra, 2016. hal-02558498

\section{HAL Id: hal-02558498 \\ https://hal.uca.fr/hal-02558498}

Submitted on 29 Apr 2020

HAL is a multi-disciplinary open access archive for the deposit and dissemination of scientific research documents, whether they are published or not. The documents may come from teaching and research institutions in France or abroad, or from public or private research centers.
L'archive ouverte pluridisciplinaire HAL, est destinée au dépôt et à la diffusion de documents scientifiques de niveau recherche, publiés ou non, émanant des établissements d'enseignement et de recherche français ou étrangers, des laboratoires publics ou privés. 


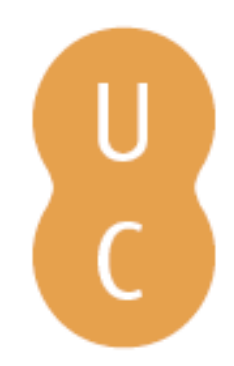

\title{
pommalina
}

\section{Euripide revisité dans Le Mystère d'Alceste de Marguerite Yourcenar}

\author{
Autor(es): $\quad$ Poignault, Rémy
}

Publicado por: Imprensa da Universidade de Coimbra; Annablume

URL

persistente: URI:http://hdl.handle.net/10316.2/40935

DOI: $\quad$ DOI:https://doi.org/10.14195/978-989-26-1298-0_29

Accessed : $\quad$ 30-Nov-2018 19:18:41

A navegação consulta e descarregamento dos títulos inseridos nas Bibliotecas Digitais UC Digitalis, UC Pombalina e UC Impactum, pressupõem a aceitação plena e sem reservas dos Termos e Condições de Uso destas Bibliotecas Digitais, disponíveis em https://digitalis.uc.pt/pt-pt/termos.

Conforme exposto nos referidos Termos e Condições de Uso, o descarregamento de títulos de acesso restrito requer uma licença válida de autorização devendo o utilizador aceder ao(s) documento(s) a partir de um endereço de IP da instituição detentora da supramencionada licença.

Ao utilizador é apenas permitido o descarregamento para uso pessoal, pelo que o emprego do(s) título(s) descarregado(s) para outro fim, designadamente comercial, carece de autorização do respetivo autor ou editor da obra.

Na medida em que todas as obras da UC Digitalis se encontram protegidas pelo Código do Direito de Autor e Direitos Conexos e demais legislação aplicável, toda a cópia, parcial ou total, deste documento, nos casos em que é legalmente admitida, deverá conter ou fazer-se acompanhar por este aviso.

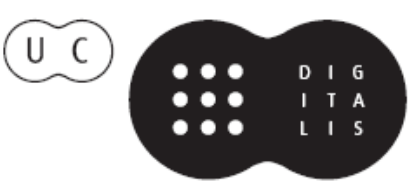




\section{O Livro do Tempo: \\ Escritas e reescritas}

\section{Teatro Greco-Latino e sua recepção II}

Maria de Fátima Silva, Maria do Céu

Fialho \& José Luís Brandão (coords.)

IMPRENSA DA UNIVERSIDADE DE COIMBRA 


\title{
Euripide REVISITÉ DANS LE MYSTĖRE D'ALCESTE DE Marguerite Yourcenar (Euripides revisited in Marguerite Yourcenar's Alcestis' Mystery)
}

\author{
RÉmy Poignault (remy.poignault1@orange.fr) \\ Université de Clermont-Ferrand - CELIS, EA 4280
}

\begin{abstract}
RÉsumé - Dans sa pièce Le Mystère d'Alceste, Marguerite Yourcenar, tout en suivant de très près la trame dramatique de l'Alceste d'Euripide en infléchit le sens en changeant les motivations du sacrifice d'Alceste et la nature du combat d'Hercule contre la mort. Entre bouffonnerie et tragédie l'auteur conserve à cette réécriture du mythe une tonalité mystique.
\end{abstract}

Mots CLÉ - Marguerite Yourcenar, Euripide, Alceste, Eros, Thanatos.

Aвstract - In her play, Le Mystère d'Alceste, Marguerite Yourcenar follows very closely the dramatic framework of Euripides' Alcestis; but she reorientates its meaning: she changes the reasons why Alcestis sacrifices her life and the nature of Hercules' fighting against death. Between buffoonery and tragedy the author retains a mystic tone while rewriting myth.

Keywords - Marguerite Yourcenar, Euripide, Alceste, Eros, Thanatos.

Lors de la Seconde Guerre Mondiale, Marguerite Yourcenar, qui s'est exilée aux États-Unis, connaît longtemps une période de désarroi et de stérilité littéraire - elle parle elle-même de l'«[e]nfoncement dans le désespoir d'un écrivain qui n'écrit pas» (CNMH, OR: 5221), ou encore d'«une œuvre au noir, où tout se défait» (Yourcenar 1980: 131). Elle rédige quelques essais sur la mythologie et la Grèce, qu'elle publie dans des revues, ou laisse inédits, comme le Cantique de l'âme libre consacré à Antinoüs, elle traduit des poèmes de Constantin Cavafy, des negro spirituals, mais aussi de la poésie grecque antique, compose elle-même quelques poèmes et surtout se lance dans l'écriture de pièces de théâtre (CNMH, OR: 523; Yourcenar 1980: 131; "Chronologie», OR: XXI-XXIII). C'est ainsi qu'elle compose Le Mystère d'Alceste en été 1942 et Électre ou la Chute des masques en été 1943, comme, d'ailleurs, inspirée d'Andersen, La Petite Sirène ${ }^{2}$. C'est, donc, en grande partie, le mythe grec et le théâtre qui permettent à Yourcenar de sortir de ce marasme

\footnotetext{
${ }^{1}$ L'abréviation $O R$ renvoie à Yourcenar 2005; CNMH désigne les «Carnets de notes de Mémoires d'Hadrien».

${ }^{2}$ Dans sa préface de Théâtre I: 137, Yourcenar donne 1942 pour la composition de La Petite Sirène, mais 1943 dans «Chronologie», OR: XXIII. C'est cette date que retient Savigneau 1990: 164, de même Barile 2007: 245.
} 
intellectuel. Yourcenar souligne l'importance, de ce point de vue, du Mystère d'Alceste, dans une lettre adressée à Maurice Jacquemont, directeur du «Studio des Champs-Élysées» qui veut et va (en 1964) monter la pièce: ce texte «a été la première œuvre écrite par moi après le choc de la guerre et du dépaysement aux États-Unis» (Yourcenar 2011: 264, 22 décembre 1962). Tout se passe comme si Alceste ressuscitait en elle l'écrivain.

Parmi les tragiques grecs, Yourcenar apprécie tout particulièrement Euripide. Dans une lettre à Jean Ballard du 4 septembre 1946, elle l'associe, d'ailleurs, étroitement à sa propre pièce, qu'elle nomme ici Alceste, en disant qu'elle souhaiterait que les Cabiers $d u$ Sud la publient «sans coupures», solution préférable «et pour Euripide, et pour moi» (Yourcenar 1995: 76).

Elle compare volontiers sa manière de procéder avec celle d'Euripide: à propos d'Électre, il est vrai, elle invoque le «réalisme d'Euripide», qui «scandalisait déjà le public d'Athènes», pour se défendre elle-même de l'«irritation» que suscite «[s]on traitement moderne et réaliste d'une légende antique» (Yourcenar 2004: 363$)^{3}$.

La maison d'édition L'Arche lui a même proposé d'écrire un ouvrage sur Euripide, mais elle a décliné l'offre à cause de l'immensité du travail que cela lui aurait demandé, préférant revenir sur son Pindare plutôt qu'ouvrir un nouveau chantier, et parce que Marie Delcourt avait déjà publié un excellent Euripide; elle suggère d'ailleurs à l'helléniste belge, par l'intermédiaire d'Alexis Curvers, de proposer une refonte de son ouvrage pour cette collection ${ }^{4}$.

Yourcenar exprime son jugement sur Euripide dans sa correspondance, comme dans ses paratextes de théâtre et dans la notice qu'elle lui consacre dans La Couronne et la Lyre. Les dialogues d'Euripide sont, à ses yeux, empreints d'un "prosaïsme exquis», qu'elle retrouve chez Cavafy, les deux poètes offrant un «réalisme anecdotique» qui «s'enveloppe de poésie», peu compréhensible pour le français, mais qui appartient à «une tradition particulièrement grecque» ${ }^{5}$.

Yourcenar, qui ne reconnaît de bonne traduction française de poésie grecque que dans «quelques vers où çà et là Racine traduit Euripide», ou dans "quelques réussites de Chénier» ${ }^{6}$, déclare aussi avoir «rêvé de traduire, ou même partiellement de récrire, pour le mettre plus à la portée du public d'aujourd'hui, l'Hippolyte d'Euripide» (Yourcenar 1995: 568)7. Mais elle publie dans La Couronne et la Lyre seulement 80 vers environ d'Hippolyte, une cinquantaine de vers

${ }^{3}$ Lettre du 22 juillet 1954 à Georges Duhamel, Yourcenar 2004: 363.

${ }^{4}$ Lettre du 3 mars 1955 à Alexis Curvers, Yourcenar 2004: 458. Marie Delcourt a traduit Euripide et est l'auteur de La Vie d'Euripide, Paris, Gallimard, 1930. Marguerite Yourcenar en possède un exemplaire: Bernier 2004, n 3604.

${ }^{5}$ Lettre du 16 mai 1963 à Jean Schlumberger, Yourcenar 2011: 412.

${ }^{6}$ Lettre du 24 mars 1963 à Étienne Coche de La Ferté, Yourcenar 2011: 376.

${ }^{7}$ Lettre du 2 octobre 1977 à Jacqueline Piatier, Yourcenar 1995: 568. 
d'Iphigénie à Aulis, une vingtaine de vers des Bacchantes, une vingtaine de vers du Cyclope et quelques fragments.

Dans sa présentation d'Euripide dans La Couronne et la Lyre, elle met l'accent sur le caractère novateur du tragique en son temps, ce qui lui a valu de ne pas connaître le succès qu'il méritait (Yourcenar 1979: 214-215); elle parle même, dans sa correspondance, d'une forme de «romantisme» d'Euripide, entendue comme «une réaction contre le classicisme antérieur» ${ }^{8}$, mais aussi comme une forme de sensibilité, «une sorte de religieux romantisme» (Th II: 93) ${ }^{9}$, sur lequel nous reviendrons. Dans La Couronne et la Lyre, elle écrit quelques années avant la parution de l'ouvrage de Jacqueline de Romilly, La modernité d'Euripide ${ }^{10}$, «[d]es trois grands dramaturges grecs, il est assurément le plus "moderne" (Yourcenar 1979: 218), mais en reconnaissant que son originalité consiste surtout à avoir poussé plus loin des effets présents chez ses prédécesseurs: sa "passion des effets mélodramatiques et des pièces à grand spectacle» a des antécédents, les "reconnaissances" de théâtre» dont il «abuse» existaient déjà chez Eschyle, le «romanesque» auquel il a recours était déjà présent chez Sophocle; les "tragédies liées"» auxquelles il renonce n'étaient pas la règle et s'il réduit le rôle du chœur «à d'anodins intermèdes lyriques, qui font déjà prévoir l'opéra moderne», «la scission entre le drame musical et la tragédie était inscrite dès le début dans le destin du drame grec» (Yourcenar 1979: 218-219). Il n'est pas davantage le premier à avoir mis en scène l'adultère, mais il y a chez lui un «double glissement vers le mélodrame et la comédie de mœurs», avec «la passion du contentieux» et du débat judiciaire (Yourcenar 1979: 220). Il s'intéresse aux personnages féminins, mais en les ramenant à un niveau moins sublime que celui qu'ils avaient chez Sophocle: «ce qui s'ébauche chez lui est ce personnage si souvent factice qu'est "la femme" dans la littérature, avec ses ruses coquettes, ses larmes faciles, et ses petites méchancetés de femme»; ainsi «[s]a Clytemnestre et son Électre ne font pas que s'affronter: elles se chamaillent» (Yourcenar 1979: 220). On pourra remarquer que si l'Alceste d'Euripide, qui ne correspond pas à cette image de la femme, conserve une grande dignité en se sacrifiant pour son époux et ses enfants, le personnage est traité dans Le Mystère d'Alceste de manière à se rapprocher de cette catégorie de femmes auxquelles le cothurne sied peu.

L'«humanitarisme» (Yourcenar 1979: 216) que Yourcenar décèle chez le tragique qui exprime «l'horreur des brutalités de la guerre, la tendre pitié à l'égard des vaincus» et des faibles (Yourcenar 1979: 215), ne doit pas tromper car il exprime une «sensibilité prodigieusement littéraire» (Yourcenar 1979: 216) et les Troyennes semblent bien avoir exalté la gloire d'Athènes et ne pas avoir «mis le

\footnotetext{
${ }^{8}$ Lettre du 18 juillet 1956 au Dr. Rudolf Allers, Yourcenar 2004: 564-565.

${ }^{9}$ L'abréviation Th II renvoie à Yourcenar 1971b.

${ }^{10}$ Romilly 1986.
} 
crime ancien en transparent sur le crime d'hier» (Yourcenar 1979: 216).

Ce qui constitue, en fait, la plus grande modernité d'Euripide, c'est que son univers n'est plus régi par des certitudes absolues: Euripide «est sans doute le premier dramaturge presque aussi complexe que la vie elle-même. Une sorte de crépuscule commence à embrumer chez lui les notions du vrai et du faux, du juste et de l'injuste» (Yourcenar 1979: 221). "L'alternance de la ferveur et du doute ajoute à son œuvre une complexité de plus»; on a du mal à cerner les contours de sa pensée, dans laquelle Yourcenar perçoit, à travers le «mysticisme» d'Hippolyte, l'«hystérie sacrée» des Bacchantes, "[1]'athéisme passionné de son Bellérophon», «l'existence de l'inquiétude religieuse chez cet homme qui semble avoir été, au sens exact du mot, un "libre penseur"» (Yourcenar 1979: 222), ce qui n'est pas sans faire penser au personnage de l'Hadrien yourcenarien. Alors que Quintilien appréciait en lui, pour l'orateur, un style proche de l'oratorium genus et tout particulièrement sa capacité à faire appel aux émotions (adfectibus) ${ }^{11}$, aux yeux de Yourcenar, Euripide va beaucoup plus loin: il est moderne dans la mesure où «[1]a notion de vie intérieure, rarissime de tout temps chez les poètes du théâtre, pointe chez lui comme un paradoxe de plus» (Yourcenar 1979: 222).

Yourcenar affirme que de ses trois pièces à sujet antique Le Mystère d'Alceste, «qui suit de très près l'étonnante tragi-comédie d'Euripide» (Yourcenar 2004: $539)^{12}$, «est la plus proche du modèle antique par l'émotion et jusqu'à un certain point par la forme. C'est du moins la seule à aborder les grands thèmes traditionnels sans leur faire subir une sorte de totale désintégration» (Th II: 99). Nous voudrions ici surtout nous interroger sur ce rendu/adaptation du ton, de l'émotion et de la forme d'Euripide chez Yourcenar.

Mais examinons d'abord la trame dramatique et les infléchissements donnés à la matière mythique par Yourcenar ${ }^{13}$. La pièce de Yourcenar, en un seul acte - et sans le chœur, dont la fonction est relayée principalement par le personnage de Georgine qui reprend aussi le rôle de la servante -, se déroule sur dix-sept scènes, qui dans l'ensemble correspondent au cheminement de la tragédie; mais sept n'ont pas d'équivalent direct chez Euripide: les scènes 4, 6 et 7 (qui introduisent des importuns, un «entrepreneur des pompes funèbres» et «le maire du village» et montrent l'étonnement de la servante Georgine devant le fait qu'Admète s'accuse du meurtre de son épouse, ce qui, toutefois, pris métaphoriquement, est bien la vérité $\left.{ }^{14}\right)$, la scène 8 , où l'arrivée de «la petite Phyllis» porteuse d'une lampe

${ }^{11}$ Quint., Inst., 10. 1. 68.

${ }^{12}$ Lettre du 21 avril 1956 à Éléonore Hirt.

${ }^{13}$ Cf. Poignault 1986; Delcroix: 1990; Poignault 1995: 285-338; Real 2007; Barile 2007.

${ }^{14}$ Dans la continuité de la modernisation du mythe depuis la Généalogie des dieux et des hommes de Boccace, Admète, chez Yourcenar, n'était pas au courant de la décision d'Alceste et, a fortiori, ne lui a pas demandé de se substituer à lui. 
considérée comme tout aussi importune par Admète est justifiée par Georgine et sert à faire le lien avec l'arrivée d'Hercule: ces scènes, en fait, comme l'arrivée des parents d'Admète à la scène 5 (au lieu du seul Phérès) avant et non plus après l'arrivée d'Hercule, inscrivent la venue d'Hercule dans ce que Yourcenar appelle «une sorte de tragi-comique ballet des Fâcheux» qui «empêchent continuellement Admète de s'abandonner à son deuil» (Th II: 101); nouvelle est la scène 11, où les bonnes veillant Alceste présentent comme le contrepoint des lamentations du chœur d'Euripide, et de celles d'Eumélos, le rôle du jeune fils d'Alceste se bornant, chez Yourcenar, à une unique réplique faite à sa mère encore vivante et qui décuple l'égoïsme inconscient de l'enfance: «Maman, tu m'avais promis de me conduire demain à la foire» (Th II: 121, sc. 3); mais les scènes rajoutées les plus importantes sont les scènes 14 et 15 où se joue, en deux temps, le combat d'Hercule contre la mort: celui-ci était seulement évoqué chez Euripide et laissé à l'imagination du spectateur, par anticipation, comme une lutte athlétique, voire une descente aux enfers (837-860), puis après-coup, mais par le biais d'une parole trompeuse, présenté à nouveau comme un agôn, puisque Héraclès prétendait avoir gagné l'inconnue voilée dans une compétition (1025-1036), avant de revenir à la première explication, mais qui n'est visiblement qu'une image: «En livrant bataille au dieu qui était son maître», «Caché près du tombeau, je l'ai saisi d'un bond» $(1140,1142)^{15}$; Yourcenar parle de ce combat contre la mort comme de «la scène à faire» (Th II: 104), mais cette scène se dédouble: le héros a d'abord à affronter la mort, qu'il parvient à faire faiblir grâce à sa volonté, puis il a à vaincre la volonté de mourir d'Alceste. Le combat, chez Yourcenar, est essentiellement moral. Maurice Delcroix a fait remarquer, en outre, que les «déambulations du cortège funèbre» ont disparu dans Le Mystère d'Alceste «de sorte que la présence de la morte pèse de tout son silence et de son immobilité sur les scènes qui précèdent son réveil» ${ }^{16}$. C'est bien le rapport de l'homme à sa finitude mortelle qui est au cœur de la méditation que provoque la pièce.

Le respect, dans ses grandes lignes, de la trame anecdotique n'empêche pas que le traitement de la légende ait subi d'importantes modifications même si Yourcenar, pour cette pièce, souligne plutôt son respect de la tradition; mais, selon une de ses formules bien connues, «le drame grec» ouvre à l'écrivain un «crédit inépuisable», puisqu'il s'agit d'une «espèce d'admirable chèque en blanc sur lequel chaque poète, à tour de rôle, peut se permettre d'inscrire le chiffre qui lui convient» (Th II: 19).

L'accent n'est plus mis sur la valeur de l'hospitalité, qui constituait un fondement essentiel de la pièce d'Euripide: si Apollon était attaché au sort d'Admète,

\footnotetext{
${ }^{15}$ Traduction Méridier 1926. Nous citerons désormais le texte dans cette traduction.

${ }^{16}$ Delcroix 1990: 84.
} 
c'était en raison de l'accueil que celui-ci avait réservé au dieu envoyé sur terre comme bouvier en châtiment pour avoir tué les Cyclopes afin de venger le foudroiement par Zeus de son fils Asclépios (1-14; 127-129) qui, selon la légende, avait employé ses talents médicaux à ressusciter des morts. Chez Yourcenar, au contraire, c'est en raison de la vénération que lui porte Admète que le dieu lui veut du bien: Apollon est ici «le profond, l'abstrait, l'indestructible soleil» (Th II: 109) et le dieu de la poésie: il est l'absolu auquel aspire le jeune homme, tentation de bien des personnages de Yourcenar, dont le tout premier, dans Le Jardin des Chimères, Icare, qui, lui, en meurt, alors qu'Admète va y trouver son salut.

L'Admète yourcenarien n'a plus rien de l'hôte qui, même aux prises avec le plus effroyable des deuils, alors qu'Héraclès, qui ne connaissait pourtant pas l'identité de celle qu'on pleurait, proposait d'aller chercher l'hospitalité ailleurs, retenait le héros (509-550), au grand étonnement du Coryphée qui y voyait une

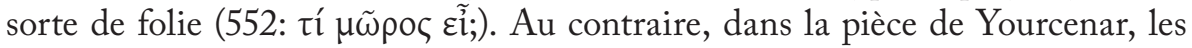
personnages qui défilent chez lui après la mort d'Alceste sont autant d'intrus qui viennent s'immiscer dans son deuil, y compris Hercule qu'Admète accueille avec une réplique très peu charitable: «Ah, c'en est trop! Dehors! Qu'on le foute à la porte!» (Th II: 132). Exit ainsi le thème de l'hospitalité. Pourquoi, alors, Hercule, une fois au courant, prend-il à cœur d'aider Admète, qui n'a consenti à l'écouter, malgré lui, que parce que Georgine, à l'opposé du Coryphée euripidien, l'a mis en garde et rappelé à la compassion envers les «mendiants»? Héraclès, chez Euripide, une fois informé, voulait selon ses propres paroles, «pour obliger Admète»

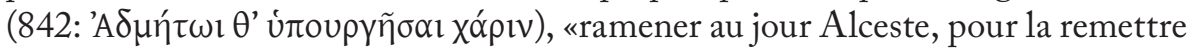

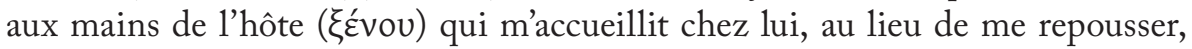
malgré le lourd malheur dont il était frappé, et qui me le cacha, noble cœur! par égard pour moi. Où trouver hospitalité plus amicale ( $\varphi \imath \lambda o ́ \xi \varepsilon v o \zeta)$ en Thessalie, et parmi les habitants de l'Hellade? Aussi ne dira-t-il pas qu'il a généreusement

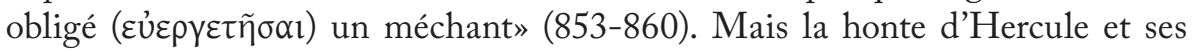
motivations ne sont pas du même type: son comportement sous le toit d'Admète est perçu par lui, qui adopte notre perspective chronologique, comme une tache morale indélébile qui ternira à jamais sa gloire: «Que ne puis-je l'effacer, cette fresque de ma vie où l'on verra éternellement un Hercule ivre dérangeant de ses hoquets le sommeil d'Alceste... Quel mot, quel geste pour réparer, pour désengager de toute cette bassesse un pur Hercule...» (Th II: 141). Hercule a le sentiment d'une faute, et même d'un péché, Yourcenar jouant des effets de l'anachronisme que favorise l'ascendance du héros: «Inspire-moi, secours-moi, Père qui es au ciel! Tu m'as fait ce que je suis, tu m'as revêtu de cette chair épaisse insensible au malheur des autres... De ce corps qui tantôt m'est un obstacle, tantôt un outil, fais ce soir l'instrument qu'il faut pour réhabiliter Hercule, pour consoler Admète, pour ramener Alceste!» (Th II: 141). C'est aussi pour son propre salut qu'il va agir en essayant de transmuer l'instrument de sa faute en tremplin salvateur. C'est son abaissement qui permettra son élévation: "Les dieux ne 
plongent pas un homme dans la boue sans le mettre en contact avec des forces vers lesquelles le reste des vivants refuse de se pencher: Hercule humilié se sent à ras de terre, à ras de sources» (Th II: 141). Il y a en lui, mutatis mutandis, quelque chose de l'Épître de saint Paul aux Philippiens (2. 8-9), qui donne cette image du Christ: «Il s'est abaissé lui-même en devenant obéissant jusqu'à mourir, et à mourir sur une croix. C'est pourquoi Dieu l'a élevé au-dessus de tout» ${ }^{17}$. Il s'agit, dans une certaine mesure, pour Hercule, d'accéder au spirituel par le corps ; mais son combat avec la mort sera plus un combat intérieur, un effort de la volonté qu'une prouesse musculaire. Il doit surmonter sa peur, accepter paradoxalement la condition humaine pour triompher du trépas: «Pour m'immuniser contre toi, je n'ai qu'à mourir» (Th II: 147); c'est également en refusant aussi bien la tentation de la mort que la tentation de la vie éternelle: "Je me contente de l'angle humain» (Th II: 148). Mais pour ramener Alceste à la vie, il ne suffit pas de faire lâcher prise à la mort, il faut aussi convaincre la jeune femme de revenir sur terre. Une fois morte, l'Alceste d'Euripide, même de retour dans le monde des vivants, gardait le silence qui sied aux ombres; mais le personnage de Yourcenar s'exprime; elle dialogue avec Hercule qui essaie de lui faire redécouvrir son identité, une identité qu'elle refuse d'abord, car sa vie antérieure ne lui plaisait pas, et l'on apprend que sa mort était une sorte de suicide. Elle veut bien alors retourner à la vie, mais pour être autre: «N'as-tu rien à me proposer d'autre que cette vieille peau dont je suis sortie, que cette robe surannée, dont je savais, dans le secret de mon cœur, qu'elle seyait mal au corps d'Alceste?...» (Th II: 152). Elle veut dépouiller la vieille Alceste, comme on se dépouille du vieil homme, mais pour tomber dans les bras du bel «homme simple, homme sain, homme aux muscles fermes» qu'est Hercule. Et l'Hercule bacchique dont l'appétit de jouissances tranchait précédemment dans la maison en deuil se met à résister à la tentation de la volupté et à défendre la vertu, comme si aussi, par un jeu d'intertextualité, Yourcenar se plaisait à prendre le contre-pied de la tragédie lyrique de Philippe Quinault, Alceste, où Alcide est amoureux de l'héroïne.

On comprend là que les motivations d'Alceste ne sont pas celles de la tradition. Dans la tragédie grecque, elle acceptait de mourir à la place de son époux, par vertu d'épouse et de mère, prenant bien soin d'assurer l'avenir de ses enfants en faisant promettre à Admète de ne pas leur donner de belle-mère: «Toi, mon époux, tu peux te flatter d'avoir pris la meilleure des femmes; vous, mes enfants, d'avoir eu la meilleure des mères» (323-325). Chez Yourcenar, quand Hercule, songeant visiblement, si l'on peut dire, à la plainte lyrique de l'Eumélos

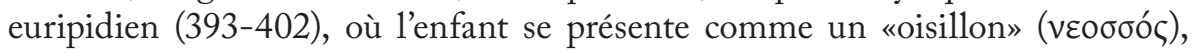
fait appel à l'instinct maternel pour attirer la morte vers la vie: «Tes enfants

${ }^{17}$ Humiliavit semetipsum factus obediens usque ad mortem, mortem autem crucis. Propter quod et Deus exaltavit illum, et donavit illi nomen quod est super omne nomen. 
t'appellent, Alceste... Tes deux enfants crient, la bouche grande ouverte, comme des oisillons au bord du nid déserté...», elle réplique «Mes deux enfants... J'ai tous les enfants morts...» (Th II: 151). Ce n'est que progressivement, grâce à sa force persuasive, qu'Hercule parvient à lui faire accepter de reprendre sa vie antérieure.

La voilà donc ressuscitée. Mais pourquoi, donc, était-elle morte? On retrouve bien des résidus de la légende: Apollon rappelle effectivement dans la scène 1 l'existence d'«une vieille malédiction familiale [qui] exige qu'à chaque génération l'amant meure pour l'amante, ou l'épouse pour l'époux» et il nous apprend qu' "[e]n secret, Alceste s'est dévouée à la mort pour ce mari plus cher que tout» (Th II: 109); la version officielle, parole divine - mais Apollon est le dieu des poètes -, exprime le mythe; Admète, qui, certes ici n'était pas au courant du rite de substitution, croit lui aussi que son épouse est morte pour lui: «Elle est morte de tendresse, morte de bonté, morte de sacrifice» (Th II: 125); «Seule, Alceste a donné à Admète son amour, son attention, sa tendresse, et enfin sa vie, qu'on ne lui demandait pas» (Th II: 127); le poète éthéré tient particulièrement à cette image idéalisée puisqu'il demande à sa femme, dans la scène où elle va mourir, de ne pas ternir son image ${ }^{18}$ : «Ne détruis pas ce chefd'œuvre, qui seul justifie ta mort! [...] ne profite pas de ces quelques instants qui nous restent pour trahir la morte, qui, elle, ne se contredira plus... Il s'agit de ta gloire ${ }^{19 ! » ~(T h ~ I I: ~ 118) ; ~ c ' e s t ~ a u s s i ~ l a ~ v e r s i o n ~ d u ~ s a c r i f i c e ~ q u e ~ r e p r e n d ~ H e r c u l e: ~}$ «Tu t'es sacrifiée pour lui... Tu t'es donnée en exemple à toutes les femmes... Tu peux rentrer dans la vie les mains pures» (Th II: 151); mais c'est une autre vérité qui sort de la bouche de la ressuscitée: «Je ne me suis pas sacrifiée... Je voulais mourir...»; Admète l'«a fait souffrir», il fut son «bourreau»; nous retrouvons là la violence des rapports amoureux de Feux ou du Coup de grâce; Alceste n'est pas morte pour Admète mais «à cause d'Admète» $(T h: 152)^{20}$. En effet, elle s'estimait mal aimée: elle, qui aurait voulu être tout pour lui, avait un rival en l'absolu qui n'était pas elle et qui constituait l'objet de la quête d'Admète. La scène entre les deux époux juste avant la mort d'Alceste nous avait, en fait, déjà avertis, avec des mots à double sens; à Admète constatant «Tu ne m'aimes plus», elle répondait: «Aime-t-on sa plaie? Chérit-on le couteau?...» (Th II: 117), où l'on pouvait voir une allusion au sacrifice, mais où la suite invite plutôt à lire que sa vie d'épouse fut pleine de désillusions; d'ailleurs, Alceste a exprimé sa jalousie par rapport aux autres femmes, mais surtout par rapport aux centres d'intérêt d'Admète, elle qui aurait voulu une transparence totale entre les deux époux: «Tu vivais

\footnotetext{
${ }^{18}$ Cf., par exemple, Poniewaz 2013: 62.

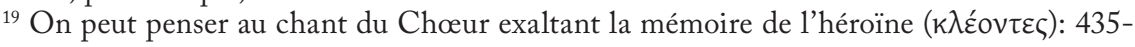
475.

${ }^{20}$ Howard 1992: 47 rapproche le destin d'Alceste de celui de la multitude des femmes qui souffrent dans leur vie conjugale.
} 
dans un palais dont $\mathrm{j}$ 'habitais tout au plus le jardin... Tu avais ton dieu, tu avais ton âme... Et la nuit, entre deux caresses, tu te levais du lit pour regarder les étoiles... Je n'ai jamais su ta pensée sur les étoiles» (Th II: 119).

Si elle accepte de revenir à la vie et à sa vie, c'est parce qu'elle apprend à transiger quand elle entend Admète regretter son manque d'attention; lorsqu'elle l'entend dire "Malheureux, qui n'as pas préféré à tout le bonheur d'Alceste...», elle l'excuse: «Il a fait de son mieux... C'est ridicule...» (Th II: 156). Il semble bien que tout soit prêt pour un dénouement heureux quand, par une image, dans sa dernière réplique, Admète associe ses deux passions en voyant le soleil levant éclairer le visage de son épouse revenue à la vie: «Dieu, tu n’es jamais aussi beau que sur une face humaine...» (Th II: 160): c'est dans le compromis entre la quête d'absolu et l'acceptation de l'humain que se trouve la voie du bonheur, car hors de l'humain il n'y a pas de salut: c'est la leçon d'Hercule dans son combat contre la mort, c'est aussi la leçon d'Alceste. C'est sans doute un élément fondamental $\mathrm{du}$ «mystère d'Alceste» qui, selon les propos mêmes de Marguerite Yourcenar, «devient pour nous un mystère d'Hercule» (Th II: 104).

Il y a là comme une invitation à réfléchir sur le titre de l'ouvrage. Il est bien connu que le statut générique de la pièce d'Euripide est ambigu. Un argument, attribué au grammairien Aristophane de Byzance, remarque que le dénouement relève de la comédie ( $\kappa \omega \mu$ pièce en quatrième position dans la série des pièces données par Euripide en 438, ce qui est la place dévolue ordinairement au drame satyrique; un ajout précise

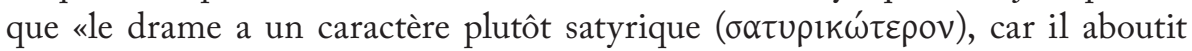
à un dénouement joyeux et agréable, contrairement à l'usage tragique» ${ }^{21}$. Si la scène avec le serviteur où Héraclès, coupe à la main et la tête couronnée, vante les plaisirs de la vie peut renvoyer à la tonalité du drame satyrique, la pièce ne comporte pas de chœur de satyres, ni un cadre champêtre, ce qui l'empêche de correspondre aux critères du genre. On a parlé de "prosatyric play", "pièce qui prend la place d'un drame satyrique». D'autre part, la pièce présente bien un élément constitutif de la tragédie, la lutte contre l'adversité ${ }^{2}$, un dénouement heureux n'étant pas incompatible avec la tragédie puisque Aristote envisage «un retournement qui conduit au bonheur ou au malheur» ${ }^{23}$.

Yourcenar, analysant la pièce d'Euripide dans son «Examen d'Alceste», revient sur son ambiguité générique en constatant: «Nous glissons sans cesse

${ }^{21}$ Cf. Méridier 1926: 49; Assaël 2015: 24, 69-73.

22 Assaël 2015: 48.

${ }^{23}$ Arist., Po. 1455b, trad. Michel Magnien, Le livre de poche classique, cité par Assaël 2015: 71. Roisman 2005: 74, considère qu'Euripide traite le sacrifice d'Alceste sur le mode tragique et l'attachement d'Admète à la vie sur le mode comique; pour Slater 2005: 92-93, la première partie de la pièce, rituel de deuil, est tragique et la seconde, rituel de mariage, plus dionysiaque. 
du plan de la tragédie à celui de la comédie romantique la plus artificielle, et de passages où l'inspiration du poète a pleinement joué à d'autres où il se débarrasse comme il peut d'un mythe auquel il ne croit plus» (Th I: 90) ${ }^{24}$. Yourcenar est au fait des débats des «érudits» qui «se sont demandés si cette pièce hybride était ou non une tragédie» (Th II: 91). Elle rapproche du drame satyrique l'ivresse d'Hercule, «la scène entre le père et le fils [qui] tourne [...] au grotesque et à la farce» (Th II: 92), mais, constate-t-elle, il manque «l'habituel chœur des Satyres» (ibid.). Et elle conclut à une «pièce incertaine» (ibid.).

Le Mystère d'Alceste n'est pas une œuvre plus facile à caractériser d'un point de vue générique. Yourcenar met en avant une sorte de dualité tonale pour répondre à ceux qui trouvent la pièce «trop traditionnelle»: «le libre mélange de poésie et de satire», qu'on trouve dans Le Mystère d'Alceste et Qui n'a pas son Minotaure?, est, en fait, en France «malgré tout assez rare» (Yourcenar 2011: $407)^{25}$. Le comique y côtoie le tragique ${ }^{26}$. Yourcenar entend recréer une tonalité hybride analogue à celle d'Euripide: «Mon but en composant cette pièce était de rénover pieusement une légende antique pour la rendre s'il se peut plus immédiatement accessible, pour en dégager d'une part l'éternelle tragi-comédie du deuil, la ronde des importuns et des indifférents toujours présente autour d'un lit funèbre, de l'autre les aspects presque liturgiques, le jeu de mort et de résurrection de ce qui est véritablement, au sens que notre Moyen Âge donnait à ce mot, un mystère, c'est-à-dire un drame sacré» (Th II: 99-100). Voilà, donc, un élément d'explication du sens du titre. Est-ce à dire toutefois que l'œuvre ait pour autant puisé au mystère médiéval? On y retrouve l'aspect religieux, le mélange du tragique avec le bouffon et le trivial, mais on y chercherait en vain, par exemple, «l'osmose entre les acteurs et le public» ${ }^{27}$, sauf à la restreindre à la maisonnée; il n'y a pas davantage de décors simultanés. C'est bien une pièce classique dans sa structure qu'écrit Yourcenar, avec unité de temps, de lieu, d'action. Mais c'est la dimension de la sacralité qui constitue le lien. Yourcenar, sans disqualifier les explications psychologiques, voit quelque chose de plus fondamental dans l'histoire d'Alceste et d'Admète, le «drame presque sacré des deux époux» (Th II: 103). Mais Alceste est aussi le mystère de la mort, pour Yourcenar qui présente la pièce comme «essentiellement une méditation, presque mystique, sur la mort» ${ }^{28}$. Yourcenar est sensible à la quête spirituelle du tragique grec, dont elle dit: «Âme inquiète, doutant de toutes les traditions, mais ouverte à

${ }^{24}$ La scène de querelle entre Phérès et Admète est considérée par Yourcenar comme «un incomparable épisode de comédie cynique» (Th I: 90). Contra: Méridier 1926: 50.

${ }^{25}$ Lettre du 29 avril 1963 à Karlheinz Braun.

${ }^{26}$ Cf., par exemple, Barile 2007: 249.

${ }^{27}$ Servet 2014: 546.

28 Lettre à Emmanuel Boudot-Lamotte du 23 mars 1945 (à paraître aux éditions Gallimard, Elyane Dezon Jones et Michèle Sarde éd.); cf. aussi Th II: 91. 
tous les mystères, le poète s'éloigne du sublime et du sacré traditionnels pour aborder le vaste domaine des intimations et des illuminations personnelles; il lui arrive d'y mettre une sorte de religieux romantisme. [...] Dans Alceste, il nous fait sentir l'entrebâillement des portes d'un autre monde. [...] Un frémissement nouveau, un pathétique inédit s'insinue chez lui dans les rapports de l'homme avec soi-même et avec les dieux» (Th II: 93). La critique a mis en lumière la tonalité religieuse de la pièce d'Euripide, «la puissance du divin» ${ }^{29}$ qui s'y exprime dans un contexte de philosophie orphico-pythagoricienne et de «subjectivisme radical» sophistique ${ }^{30}$; Alceste passerait par une forme de mort pour accéder à la plénitude de l'être et Héraclès ferait figure d'initiateur, remettant Alceste à Admète pour qu'il s'unisse à elle dans un rituel mystique qui prend les formes du mariage traditionnel.

Le Mystère d'Alceste conserve, au-delà du mystère médiéval, quelque chose du mystère au sens antique et présente une dimension mystique qui remonte aux premiers temps où «un souci d'immortalité paraît avoir reflué sur le monde, une inquiétude, un espoir, l'idée que la mort n'est pas une fin, mais peut-être un commencement, une naissance ou un voyage, une épreuve que les héros et les purs sont capables de surmonter et de faire surmonter à d'autres [...]» (Th II: 86) ${ }^{31}$. L'initiateur, comme chez Euripide, est toujours Hercule, un Hercule qui prend volontiers une coloration chrétienne, dans les prières qu'il adresse à son "Père qui es au ciel» (Th II: 141), comme dans son sens de la faute; et il n'est pas anodin que quand il la ramène à la vie Alceste croie voir en un «espalier de pêches mûres» «un squelette... Des clous dans des bras tendus... Un mort crucifié...» (Th II: 154); Hercule a une dimension christique, en même temps qu'il apparaît comme une sorte de soldat de la justice; mais c'est par son aspect résolument humain qu'il parvient à vaincre la mort, ramener Alceste à la vie et se laver lui-même de sa faute. Il permet, en outre, au couple d'Alceste et d'Admète de se retrouver sur des bases nouvelles: le renoncement à la tentation de l'absolu (absolu de la poésie dans un cas, absolu de l'amour dans l'autre) pour être attentif à l'autre; la «résurrection» d'Alceste est, en fait, un renoncement à la tentation du suicide, à laquelle cèdent la Sappho de Feux, «cramponnée à sa mort comme à un promontoire»(OR: 1137), ou Antinoüs «enlisé par la boue» du Nil (OR: 440).

\footnotetext{
${ }^{29}$ Assaël 2015: 90.

${ }^{30}$ Assaël 2015: 96-105.

${ }^{31}$ Lombardi 2007: 235-236, y voit aussi le mystère psychique de la coniunctio problématique du couple.
} 


\section{Bibliographie}

Assaël, J. (2015), «Euripide, Alceste», in Silves grecques 2015-2016. Neuilly: Atlande.

Barile, L. (2007), «Il Mistero di Alcesti», in Pinzuti, E. (ed.), Marguerite Yourcenar sulle tracce "des accidents passagers". Roma, Bulzoni Editore: 243260.

Bernier, Y. (2004), Inventaire de la bibliothèque de Marguerite Yourcenar. Petite Plaisance. Clermont-Ferrand: SIEY.

Delcroix, M. (1990), «Dramaturgie du Mystère d'Alceste : la scène à faire», Bulletin de la Société Internationale d'Études Yourcenariennes 7, déc.: 81-97.

Howard, J. E. (1992), From violence to vision. Sacrifice in the works of Marguerite Yourcenar. Carbondale and Edwardsville, Southern Illinois University Press: 42-75.

Kincaid, M. J. (2005), Yourcenar dramaturge. Microcosme d'une œuvre. New York: Peter Lang.

Lombardi, M. (2007), «Pia e Alceste. Il teatro della coppia», in Pinzuti, E. (ed.), Marguerite Yourcenar sulle tracce "des accidents passagers". Roma, Bulzoni Editore: 223-241.

Méridier, L. (ed.) (1926), Euripide, Le Cyclope, Alceste, Médée, Les Héraclides. Paris: Les Belles Lettres [rééd.: 2003]

Poignault, R. (1986), «Le Mystère d'Alceste : rénovation et métamorphose du mythe», Il Confronto Letterario, suppl. al n 5: 69-80.

Poignault, R. (1995), L'Antiquité dans l'œuvre de Marguerite Yourcenar. Littérature, mythe et histoire, I. Bruxelles: coll. "Latomus".

Poniewaz, K. A. (2013), «Alcestes and her sisters: feminism, sorority and tragedy in Yourcenar and Simone's Alcestes», Women in French Studies 21: 56-67.

Real, E. (2007), «El Misterio de Alcestes de Marguerite Yourcenar: reinterpretación del mito griego», in Bañuls, J. V., De Martino, F., Morenilla, C. (eds.), El teatro greco-latino y su recepción en la tradición occidental 2. Bari, Levante Editori: 711-728.

Roisman, H. M. (2005), «The Cyclops and the Alcestis: tragic and the absurd», in Harrison, G. W. M. (ed.), Satyr Drama. Tragedy at Play. Swansea, The Classical Press of Wales: 67-82.

Romilly, J. de (1986), La modernité d'Euripide. Paris: PUF.

Savigneau, J. (1990), Marguerite Yourcenar. L'invention d'une vie. Paris: Gallimard.

Servet, P. (2014), «Mystère», in Neiva, S., Montandon, A. (eds.), Dictionnaire raisonné de la caducité des genres littéraires. Genève, Droz: 543-558. 
Slater, N. W. (2005), «Nothing to do with satyrs? Alcestis and the concept of prosatyric drama», in Harrison, G. W. M. (ed.), Satyr Drama. Tragedy at Play. Swansea, The Classical Press of Wales: 83-101.

Yourcenar, M. (1944a), «Mythologie», Lettres françaises (Buenos Aires) 11, 1er janv.: 41-46.

Yourcenar, M. (1944b), «Mythologie II - Alceste», ibid. 14, 1er oct.: 33-40.

Yourcenar, M. (1945), «Mythologie III - Ariane - Électre», ibid. 15, 1er janv.: 35-45.

Yourcenar, M. (1971a), Théâtre I. Paris: Gallimard.

Yourcenar, M. (1971b), Théâtre II. Paris: Gallimard.

Yourcenar, M. (1979), La Couronne et la Lyre. Paris: Gallimard.

Yourcenar, M. (1980), Les Yeux ouverts. Paris: Le centurion.

Yourcenar, M. (1995), Lettres à ses amis et quelques autres. Sarde, M., Brami, J. (eds.). Paris: Gallimard.

Yourcenar, M. (2004), D’Hadrien à Zénon. Correspondance 1951-1956. Gaudin, C., Poignault, R. (eds.). Paris: Gallimard.

Yourcenar, M. (2005), Euvres romanesques. Paris: Gallimard, coll. "Bibliothèque de la Pléiade".

Yourcenar, M. (2011), «Persévérer dans l'être». Correspondance 1961-1963. Brami, J., Poignault, R. (eds.). Paris: Gallimard. 